\title{
Integral Solution of the Interface Profile of Grain Boundary Grooving by Surface Diffusion
}

\author{
Caifang Wang, ${ }^{1}$ Hui Xing, ${ }^{2}$ Fengping Yao, ${ }^{3}$ Changle Chen, ${ }^{2}$ \\ Jianyuan Wang, ${ }^{2}$ and Fei Fan ${ }^{2}$ \\ ${ }^{1}$ Department of Mathematics, Shanghai Maritime University, Shanghai 201306, China \\ ${ }^{2}$ Shaanxi Key Laboratory for Condensed Matter Structure and Properties, Northwestern Polytechnical University, \\ Xi'an 710072, China \\ ${ }^{3}$ Department of Mathematics, Shanghai University, Shanghai 200444, China
}

Correspondence should be addressed to Changle Chen, chenchl@nwpu.edu.cn

Received 23 June 2012; Revised 14 October 2012; Accepted 15 October 2012

Academic Editor: Pavel Lejcek

Copyright (c) 2012 Caifang Wang et al. This is an open access article distributed under the Creative Commons Attribution License, which permits unrestricted use, distribution, and reproduction in any medium, provided the original work is properly cited.

In polycrystalline thin films, formation of grain boundary grooving is investigated widely due to its effects on their properties. Infinite series solution of the interface of grain-boundary grooving by surface diffusion has been obtained. In this paper, with Fourier transform, a novel integral solution of the profile of grain boundary grooving is derived. Numerical examples are taken to test the performance of the integral solution. According to the numerical examples, we find that the novel solution is in accordance with the infinite series solution.

\section{Introduction}

In polycrystalline thin films, grain boundary grooving through the thickness of the film is a common failure mode that strongly affects their properties. Formation and development of grain boundary grooving have received a great deal of attention over decades [1-6]. The grooving forms and develops at the point of intersection when the grain boundary ends at a free boundary in order to reduce the total free energy. In absence of the external potential field, the grooving profile migrates via surface diffusion. Figure 1 shows the AFM image of grain boundary grooving of $\mathrm{BiFeO}_{3} / \mathrm{LaNiO}_{3}$ thin film. Mass transport by surface diffusion is driven by the curvature Laplacian. For convex surface, mass flows from high curvature parts to low parts, while in reverse for concave surfaces. The theory of the capillary-driven shape evolution was first provided by Mullins [1], and this kind of problem hence is socalled Mullins problem. In his study, he considered that the grooving is symmetric between the two grains in the small slope approximation. Assume that the surface energy is isotropic and the surface flux can be expressed as

$$
J_{s}=-\frac{D_{s} \gamma \Omega n_{s}}{k T} \nabla_{s} \kappa
$$

where $\nabla_{s} \kappa$ is the surface curvature gradient, $D_{s}$ is the coefficient of surface diffusion, $s$ is the arc length of the interface profile, $\gamma$ is the isotropic surface energy, $\Omega$ is the atomic volume, $n_{s}$ is the number of mobile atoms in the surface layer per unit, and $k T$ is with its usual meaning. Therefore the surface normal speed can be written as

$$
V_{n}=\frac{D_{s} \gamma \Omega^{2} n_{s}}{k T} \nabla_{s}^{2} \kappa
$$

Defining $y=y(x, t)$ as the interface profile and using $\kappa=$ $-y^{\prime \prime}\left(1+y^{\prime}\right)^{-2 / 3}$, we could get

$$
y_{t}=-B \partial_{x}\left[\left(1+Y^{\prime 2}\right)^{1 / 2} \partial_{x}\left(1+y^{\prime}\right)^{-2 / 3}\right],
$$

where $B=D_{s} \gamma \Omega^{2} n_{s} / k T$. In small slope approximation, it is reduced to

$$
y_{t}=-B y^{\prime \prime \prime \prime} .
$$

Wong et al. [7] have shown that the film profiles at different times are found to be self-similar. The wedge contact angle will change at initial time from wedge angle $\alpha$ to the equilibrium $\beta$. Therefore, the partial differential equation can be converted into an ordinary differential equation before 


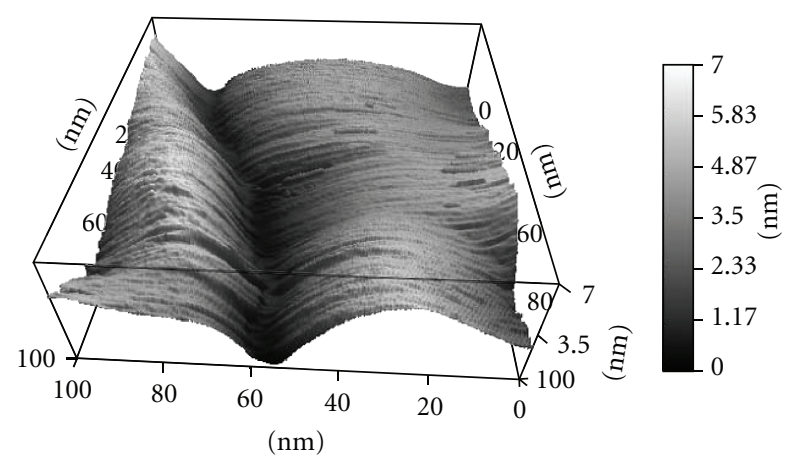

FIGURE 1: AFM image of grain boundary grooving of $\mathrm{BiFeO}_{3}$ $/ \mathrm{LaNiO}_{3}$ thin film.

solving it by the integral method. In this paper, we mainly seek the integral solution of (4) with proper initial and boundary conditions. The rest of the paper is organized as follows. In Section 2, we derive the main integral solution of the interface profile with Fourier transform. Then we change the integral solution into normalized solution with some variable substitution. In Section 3, we take numerical experiments to test the novel integral solution. In Section 4, we give the conclusion of this paper.

\section{Integral Solution}

In the following, we consider the symmetric case of the profile $y(x, t)$. Since the symmetry is about $y$ axis, we only consider the profile $y(x, t)$ for $x>0$. As in [1], we assume that the initial surface is flat. Also, we assume that at the groove root, the flux is zero and there is a fixed dihedral angle. Hence, the groove profile $y(x, t)$ satisfies the following initial boundary problem:

$$
\begin{gathered}
y_{t}+B y^{\prime \prime \prime \prime}=0, \quad x>0, \\
y(x, 0)=0, \\
y^{\prime}(0, t)=m, \\
y^{\prime \prime \prime}(0, t)=0,
\end{gathered}
$$

where $m$ is the slope related to the dihedral angle enclosed by the surface.

2.1. Main Solution. According to the theories of partial differential equations and Fourier transform, we can get the integral solution of the initial boundary problem (5) as follows:

$$
\begin{aligned}
y(x, t)= & m x-\frac{1}{2 \pi} \\
& \quad \times \int_{0}^{\infty} \int_{-\infty}^{\infty} e^{-B \xi^{4} t} \\
& \times(\cos \xi(x-y)+\cos \xi(x+y)) \\
& \cdot m y \mathrm{~d} \xi \mathrm{d} y .
\end{aligned}
$$

In the following, we use three steps to derive the integral solution (6).

Let $z(x, t)=m x-y(x, t)$. Then we only need to seek the solution $z(x, t)$ of the following problems:

$$
\begin{gathered}
z_{t}+B z^{\prime \prime \prime \prime}=0, \quad x>0, \\
z(x, 0)=m x, \\
z^{\prime}(0, t)=0, \\
z^{\prime \prime \prime}(0, t)=0 .
\end{gathered}
$$

Step 1. We first consider the solution of

$$
\begin{gathered}
w_{t}+B w^{\prime \prime \prime \prime}=0, \quad-\infty<x<\infty, \\
w(x, 0)=g(x) .
\end{gathered}
$$

By taking Fourier transform with respect to $x$, we can obtain the solution of (11)

$$
\begin{aligned}
w(x, t) & =\frac{1}{2 \pi} \int_{-\infty}^{\infty} g(y) \mathrm{d} y \int_{-\infty}^{\infty} e^{-B \xi^{4} t} e^{-i \xi(x-y)} \mathrm{d} \xi \\
& =\frac{1}{2 \pi} \int_{-\infty}^{\infty} g(y) \mathrm{d} y \int_{-\infty}^{\infty} e^{-B \xi^{4} t} \cos \xi(x-y) \mathrm{d} \xi .
\end{aligned}
$$

Since a Schwartz function's Fourier transform is still a Schwartz function, we deduce that

$$
\int_{-\infty}^{\infty} e^{-B \xi^{4} t} e^{-i \xi(x-y)} \mathrm{d} \xi
$$

is a Schwarz function for the variable $y$, which makes the double integral sense in (12) when $g(x)$ is a polynomial function. In fact, we can refer to the paper [8] for further discussions of elementary theory of (11).

Step 2. It is easy to verify that

$$
\begin{aligned}
z(x, t)= & \frac{1}{2 \pi} \\
& \quad \times \int_{0}^{\infty} \int_{-\infty}^{\infty} e^{-B \xi^{4} t} \\
& \times(\cos \xi(x-y)+\cos \xi(x+y)) \\
& \cdot m y \mathrm{~d} \xi \mathrm{d} y
\end{aligned}
$$

satisfies (7)-(10). In fact, it is trivial to prove that $z(x, t)$ satisfies (7), (9), and (10). Moreover, we can verify that $z(x, t)$ also satisfies (8) by choosing

$$
g(x)= \begin{cases}m x, & x>0 \\ -m x, & x<0\end{cases}
$$


Step 3. Finally, we can obtain the solution of (5)

$$
\begin{aligned}
y(x, t)= & m x-z(x, t) \\
= & m x-\frac{1}{2 \pi} \\
& \times \int_{0}^{\infty} \int_{-\infty}^{\infty} e^{-B \xi^{4} t} \\
& \times(\cos \xi(x-y)+\cos \xi(x+y)) \\
& \cdot m y \mathrm{~d} \xi \mathrm{d} y .
\end{aligned}
$$

2.2. Normalized Solution. Let $\bar{x}=x(B t)^{-1 / 4}, \bar{y}=y(B t)^{-1 / 4}$ and $\tau=\xi(B t)^{1 / 4}$. With the variable substitution, the function $z(x, t)$ can also be expressed as

$$
\begin{gathered}
z(\bar{x}, t)=\frac{m(B t)^{1 / 4}}{2 \pi} \\
\quad \times \int_{0}^{\infty} \int_{-\infty}^{\infty} e^{-\tau^{4}} \\
\quad \times(\cos \tau(\bar{x}-\bar{y})+\cos \tau(\bar{x}+\bar{y})) \\
\cdot \bar{y} \mathrm{~d} \tau \mathrm{d} \bar{y} .
\end{gathered}
$$

Let

$$
h(u)=\int_{-\infty}^{\infty} e^{-\tau^{4}} \cos \tau u \mathrm{~d} \tau .
$$

It is the Fourier transform of a Schwartz function $e^{-\tau^{4}}$. According to the properties of Fourier transform, $h(u)$ is still Schwartz function and $z(\bar{x}, t)$ is integrable. With simple calculation, we arrive at

$$
\begin{aligned}
z(\bar{x}, t)=\frac{m(B t)^{1 / 4}}{2 \pi} \times & {\left[2 \bar{x} \int_{0}^{\bar{x}} h(u) \mathrm{d} u\right.} \\
& \left.\quad-2 \int_{0}^{\bar{x}} h(u) u \mathrm{~d} u+2 \int_{0}^{\infty} h(u) u \mathrm{~d} u\right] .
\end{aligned}
$$

Finally, we get the normalized solution of the initial boundary problem (5)

$$
\begin{aligned}
& y(\bar{x}, t)= m(B t)^{1 / 4} \bar{x}-z(\bar{x}, t) \\
&=\frac{m(B t)^{1 / 4}}{\pi}\left[\pi \bar{x}-\bar{x} \int_{0}^{\bar{x}} h(u) \mathrm{d} u\right. \\
&\left.\quad+\int_{0}^{\bar{x}} h(u) u \mathrm{~d} u-\int_{0}^{\infty} h(u) u \mathrm{~d} u\right] .
\end{aligned}
$$

\section{Numerical Test}

In this section, we take numerical experiments to show the performance of our integral solution of the interface profile of grain boundary grooving. All the numerical tests are implemented on a Dell desktop 4311s (Intel Core2 Duo CPU E7400 $2.8 \mathrm{GHz}$ with $2.00 \mathrm{~GB}$ RAM). The software Matlab is used to calculate all the numerical solutions.
TABLE 1: Number of terms to truncated error for different $u$.

\begin{tabular}{ccccccccc}
\hline & $u$ & 0.1 & 0.5 & 1 & 2 & 5 & 10 & 20 \\
\hline \multirow{4}{*}{$N \quad(e=1 e-7)$} & 2 & 3 & 5 & 7 & 13 & 25 & 54 \\
& $(e=1 e-8)$ & 2 & 4 & 5 & 8 & 14 & 26 & 55 \\
& $(e=1 e-10)$ & 3 & 5 & 6 & 9 & 16 & 28 & 57 \\
& $(e=1 e-12)$ & 3 & 5 & 7 & 10 & 18 & 31 & 60 \\
\hline
\end{tabular}

3.1. Numerical Calculation of $h(u)$. We first calculate Schwartz function $h(u)$. To facilitate the calculation, we expand the function $\cos \tau u$ in (18) in a power series and integrate term by term. Then we get

$$
h(u)=\int_{-\infty}^{\infty} e^{-\tau^{4}} \cos \tau u \mathrm{~d} \tau=\frac{1}{2} \sum_{n=0}^{+\infty}(-1)^{n} \frac{\Gamma((2 n+1) / 4)}{(2 n) !} u^{2 n}
$$

where $\Gamma(u)$ is the gamma function. Since $h(u)$ is a series form, we use finite terms

$$
h_{2 N}(u)=\frac{1}{2} \sum_{n=0}^{N}(-1)^{n} \frac{\Gamma((2 n+1) / 4)}{(2 n)} u^{2 n}
$$

to approximate $h(u)$ in the numerical tests. The truncated remainder for $h(u)$ is

$$
\begin{aligned}
R_{2(N+1)}(u) & =\left|\frac{h^{(2 N+2)}(\xi)}{(2 N+2) !} u^{2 N+2}\right| \quad \xi \in(0, u) \\
& \leq \frac{\Gamma((2 N+3) / 4)}{(2 N+2) !} u^{2 N+2} .
\end{aligned}
$$

We exam the dependence of truncated error and number of terms for different $u$ in $h(u)$ with decreasing truncated error. Table 1 illustrates the details and Table 2 lists the values of $h_{2 N}(u)$ and $u h_{2 N}(u)$ when truncated error $e=1 e-7$. It is shown that the number of terms used exploded for large $u$ as truncated error decreased. However, we do not have to calculate the value of $h(u)$ for those $u$ far away from zero since $h(u)$ is a Schwarz function.

3.2. Integral Solution. In the following, we provide the numerical result of our integral solution. $h(u)$ used in the integral solution is approximated by $h_{120}(u)$. The part of infinite integration $\int_{0}^{\infty} h(u) u \mathrm{~d} u$ is truncated to finite integral over the interval from zero to 20 . To calculate numerical integration, trapezium integral formula is applied. Since the parameter $(B t)^{1 / 4}$ only impacts the scale of the solution $y(\bar{x}, t)$, here we select $(B t)^{1 / 4}=1$. We plot the normalized profile $y / m$ in Figure 2. To test the performance of our integral solution, we also compare the integral solution with Mullins' series solution [1] at $|\bar{x}| \leq 5$. Here Mullins solution of the interface profile is displayed as

$$
y(\bar{x}, t)=m(B t)^{1 / 4} \sum_{n=0}^{+\infty} a_{n} \bar{x}^{n}
$$


TABLE 2: Values of $h_{2 N}(u)$ and $u h_{2 N}(u)\left(e 1=10^{-7}\right)$.

\begin{tabular}{lccccccc}
\hline$u$ & 0.1 & 0.5 & 1 & 2 & 5 & 10 & 20 \\
$N$ & 2 & 3 & 5 & 7 & 13 & 54 & 25 \\
$h_{2 N}(u)$ & 1.80974330 & 1.73738665 & 1.52470975 & 0.85205558 & -0.17151472 & -0.00269424 & -0.00000168 \\
$u h_{2 N}(u)$ & 0.18097433 & 0.86869332 & 1.52470975 & 1.70411115 & -0.85757362 & -0.02694244 & -0.00003369 \\
\hline
\end{tabular}

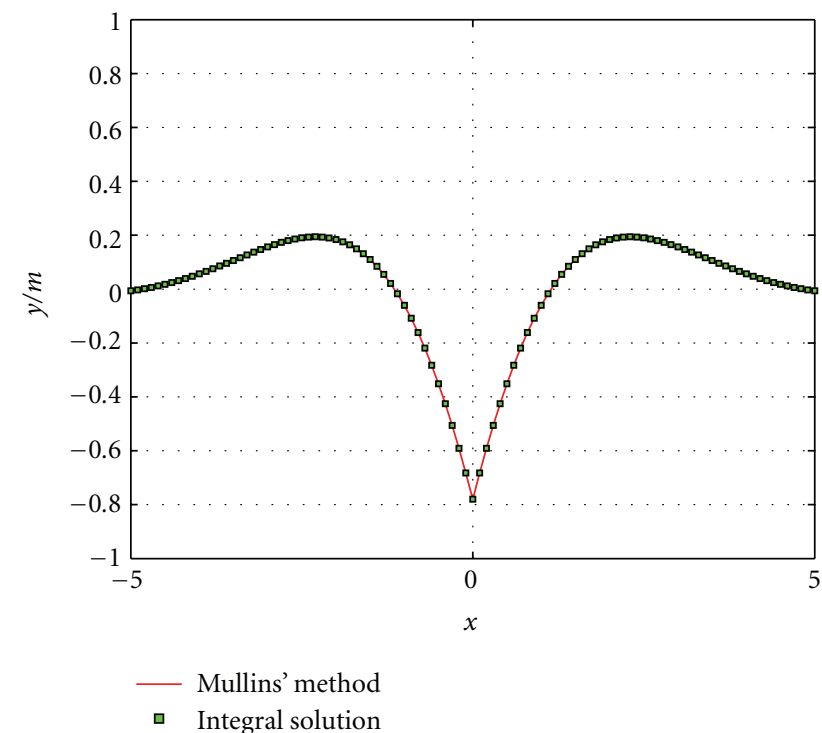

FIGURE 2: Normalized profile shape due to volume diffusion.

where $a_{n}$ is calculated by the recursion relation

$$
\begin{gathered}
a_{n+4}=a_{n} \frac{n-1}{4(n+1)(n+2)(n+3)(n+4)}, \\
a_{0}=-\frac{1}{2^{1 / 2} \Gamma(5 / 4)}, \quad a_{1}=1, \quad a_{2}=-\frac{1}{2^{3 / 2} \Gamma(3 / 4)}, \quad a_{3}=0 .
\end{gathered}
$$

Figure 2 displays the comparison results. Solid line is Mullins' solution and dash line is our integral solution. Monitoring the evolution of the depth and the width of the groove profile, we find that our solution agrees very well with Mullins' solution according to the figure.

Here we have not compared the groove profile at large $\bar{x}$ because both solutions can easily become unstable due to the limited machine precision and the truncation. For Mullins' solution, we plot the profile between $|\bar{x}| \leq 15$ with different truncation of the infinite series in Figure 3. The truncation of the infinite series is chosen as $n=25,26,27,28,41,42,43,44$, respectively. From Figure 3, we find that for $n=25,26,41$, and 42, the curves of Mullins's solution grow to positive infinity and for $n=27,28,43$, and 44 , the curves grow to negative infinity. This phenomenon appears in a fourterm cycle, and it is in agreement with the coefficients of the infinite series in (25) and (26). However, for our integral

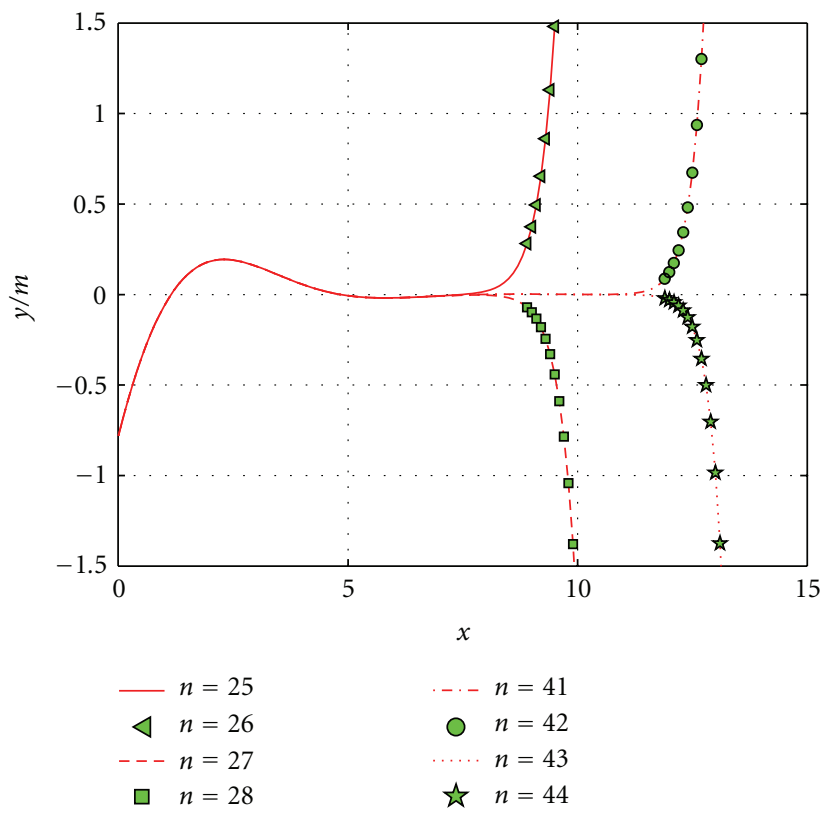

Figure 3: Normalized profile shape due to volume diffusion.

solution, with the properties of the Fourier transform, we have

$$
\begin{aligned}
\int_{0}^{+\infty} h(u) \mathrm{d} u & =\frac{1}{2} \int_{-\infty}^{+\infty} h(u) \mathrm{d} u \\
& =\pi\left(\frac{1}{2 \pi} \int_{-\infty}^{+\infty} \cos (u \cdot 0) \mathrm{d} u \int_{-\infty}^{+\infty} e^{-\tau^{4}} \cos \tau u \mathrm{~d} \tau\right) \\
& =\pi e^{-0^{4}}=\pi .
\end{aligned}
$$

Hence the groove profile $y(\bar{x}, t) \rightarrow 0$ at a fixed time when $\bar{x} \rightarrow+\infty$. Since $h(u)$ is a Schwarz function, we can easily conclude that $y(\bar{x}, t)$ is stable with the properties above, and we do not have to examine the profile at large $\bar{x}$.

3.3. Properties of Integral Solution. At last, we conduct some properties of our integral solution (20). We compute

$$
\frac{\mathrm{d} y}{\mathrm{~d} \bar{x}}=\frac{m(B t)^{1 / 4}}{\pi}\left[\pi-\int_{0}^{\bar{x}} h(u) \mathrm{d} u\right]
$$

and find that the maximum of the surface is at the first occurrence of $\bar{x}$ with

$$
\int_{0}^{\bar{x}} h(u) \mathrm{d} u=\pi .
$$




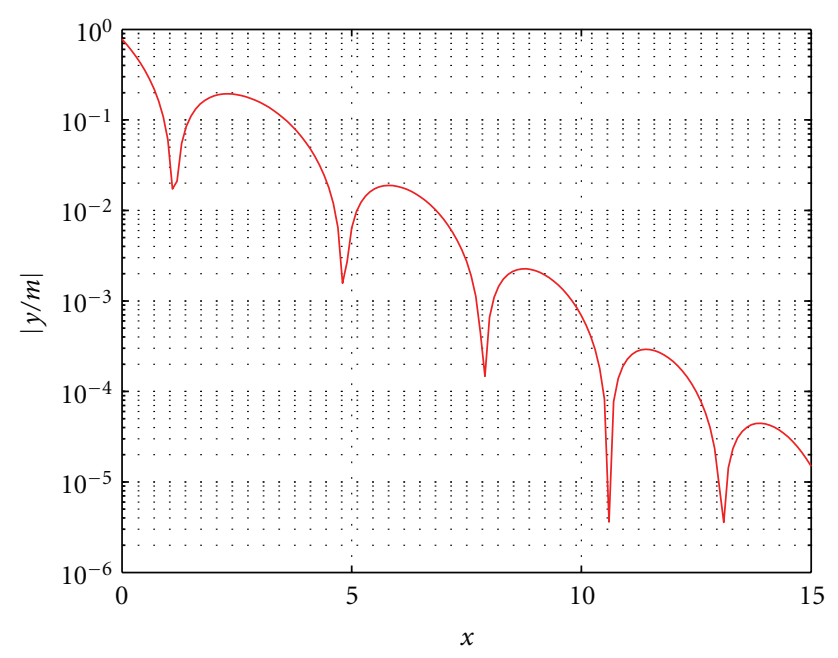

FIgURE 4: The spatial oscillation of the groove profile.

Combined with the data in Figure 2, the maximum of the surface is bout $y(2.3)=0.194$. The depth of the groove measured in $y$ direction from the maximum of the surface to the grain boundary is given by

$$
d=0.9738 m(B t)^{1 / 4},
$$

whereas the separation of the two maxima is

$$
s=4.6(B t)^{1 / 4} \text {. }
$$

From these expressions, we have the time independent ratio

$$
\frac{s}{d}=4.7236 m \text {. }
$$

The spatial oscillation of the groove profile at a fixed time is shown in Figure 4. The solution shows a spatial oscillation with a rapid exponential damping in the amplitude. Beyond this, we can also get the interval between the groove root and the beginning of the tail from the figure.

The curvature of the groove profile $y(x, t)$ is defined as $K=\left|y^{\prime \prime}\right| /\left(1+y^{\prime 2}\right)^{3 / 2}$. However, in small slope approximation, we assume that $\left|y^{\prime}\right| \ll 1$. Hence, the curvature of the normalized profile here is $K=\left|y^{\prime \prime}\right|=h(\bar{x})$. The result is plotted in Figure 5. All these properties are in agreement with Mullins' solution.

\section{Conclusion}

In this work, we mainly seek a novel solution of the interface profile of grain-boundary grooving by surface diffusion with small slope approximation. With Fourier transform theory and properties of partial differential equation, we derive an integral solution (6) for the Mullins problem. Taking variable substitution, we get the normalized solution (20). According to the numerical tests, we find that our solution is identical with Mullins' solution. The rates of growth of the depth and width remain proportional to $(B t)^{1 / 4}$. Besides, we can get the properties of the groove profile $y(\bar{x}, t)$ with theoretical analysis, and these properties are no longer affected by the machine precision and the truncation of infinite series.

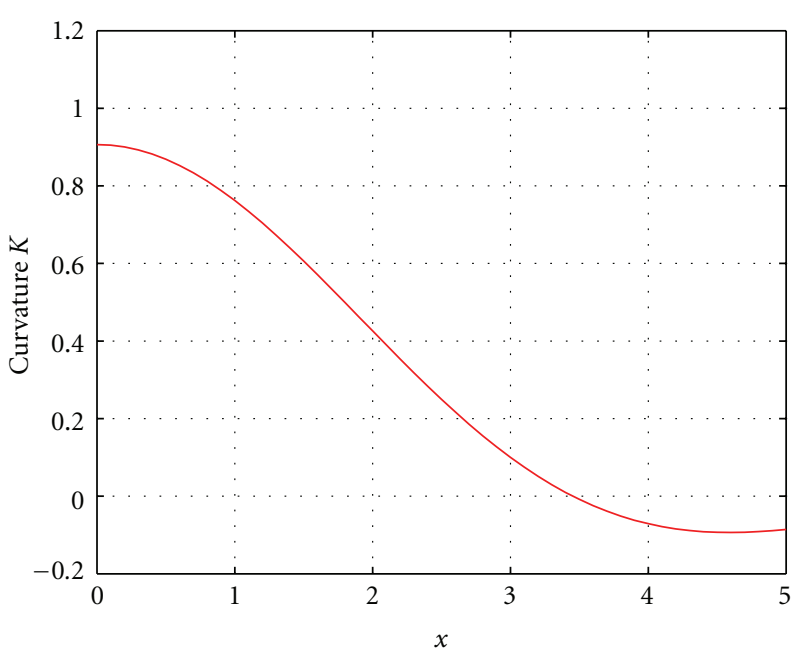

FIGURE 5: The curvature of the groove profile.

\section{Acknowledgments}

C. Wang is supported by Science and Technology Program of Shanghai Maritime University no. 20110054. H. Xing, C. Chen and F. Fan are supported by the National Natural Science Foundation of China Grant no. 61078057 and 50702046.

\section{References}

[1] W. W. Mullins, "Theory of thermal grooving," Journal of Applied Physics, vol. 28, no. 3, p. 333, 1957.

[2] W. W. Mullins, "Grain boundary grooving by volume diffusion," Transactions of the Metallurgical Society of the AIME, vol. 218, pp. 354-361, 1960.

[3] H. Zhang and H. Wong, "Coupled grooving and migration of inclined grain boundaries: regime I," Acta Materialia, vol. 50, pp. 1983-1994, 2002.

[4] M. Bouville, C. Dongzhi, and D. J. Srolovitz, "Grain-boundary grooving and agglomeration of alloy thin films with a slowdiffusing species," Physical Review Letters, vol. 98, no. 8, Article ID 085503, 2007.

[5] M. Bouville, "Effect of grain shape on the agglomeration of polycrystalline thin films," Applied Physics Letters, vol. 90, no. 6, Article ID 061904, 3 pages, 2007.

[6] L. Klinger, "Surface evolution in two-component system," Acta Materialia, vol. 50, no. 13, pp. 3385-3395, 2002.

[7] H. Wong, M. J. Miksis, P. W. Voorhees, and S. H. Davis, "Capillarity driven motion of solid film wedges," Acta Materialia, vol. 45, no. 6, pp. 2477-2484, 1997.

[8] F. Yao, "Schauder estimates for parabolic equation of biharmonic type," Applied Mathematics and Mechanics, vol. 28, no. 1, pp. 1503-1516, 2007. 

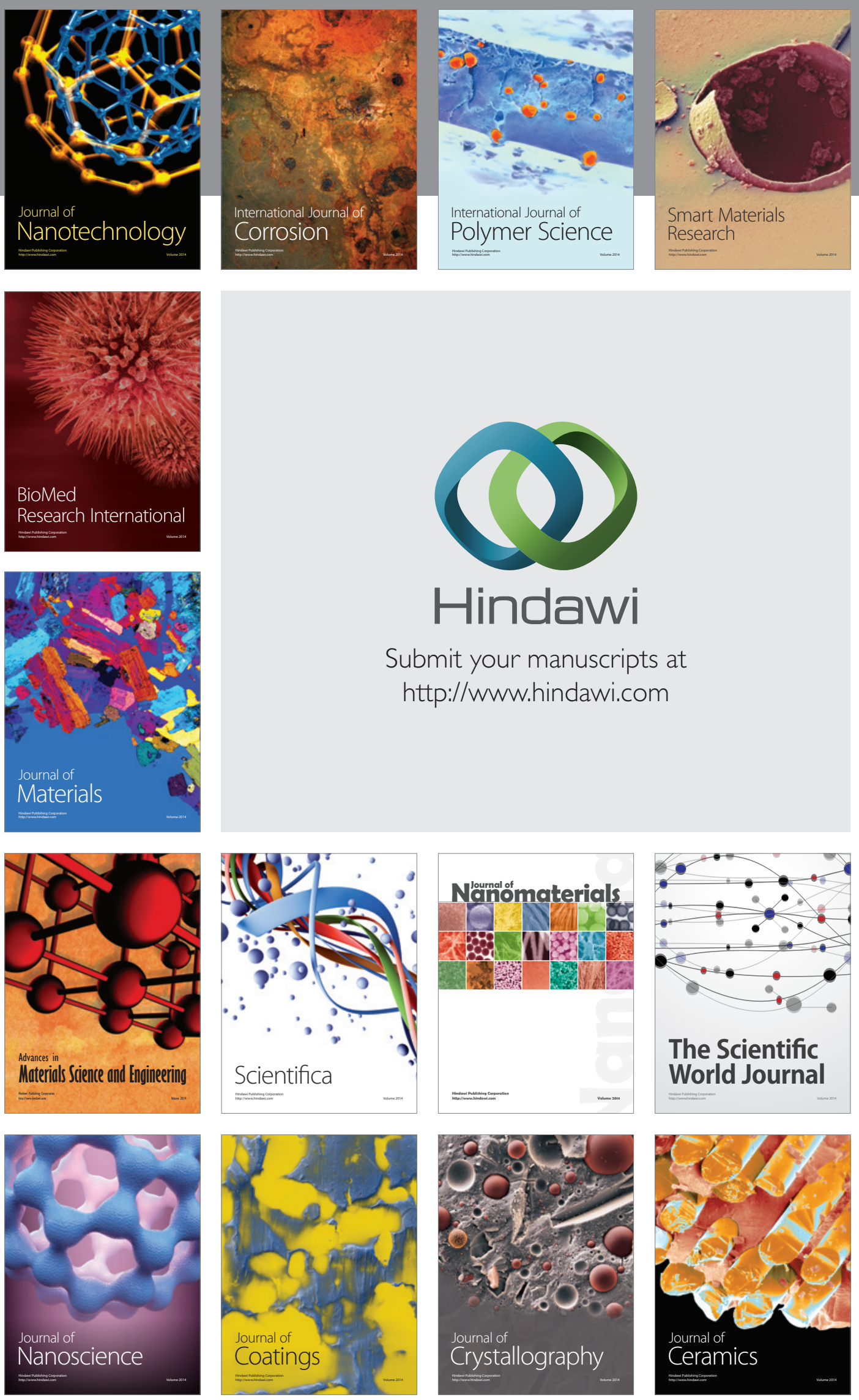

The Scientific World Journal

Submit your manuscripts at

http://www.hindawi.com

\section{World Journal}

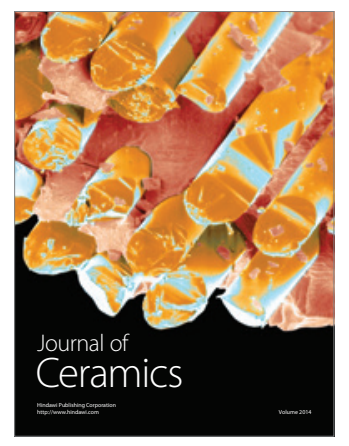

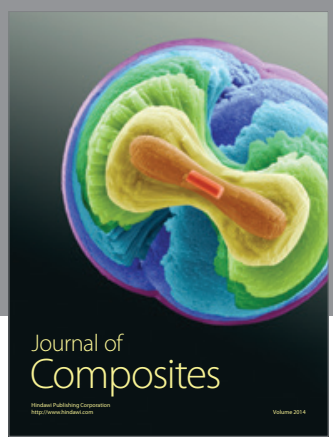
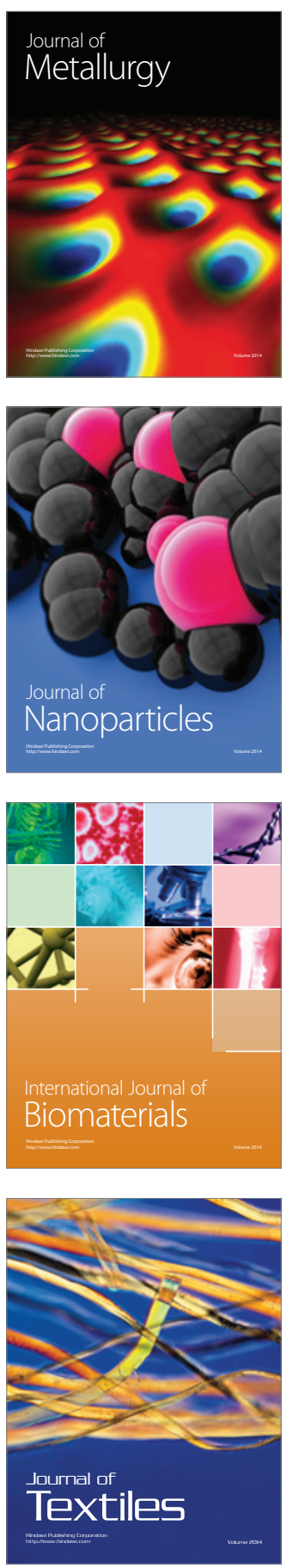